\title{
Effets pléiotropiques de l'hydroxyurée
}

Les hémoglobinopathies, de par leur sélection dans les zones d'endémie palustre, sont les plus fréquentes des maladies génétiques. Bien connues maintenant, elles suscitent d'intenses recherches d'abord thérapeutique. Des résultats spectaculaires ont certes été obtenus par la greffe de moelle; celle-ci est cependant d'usage limité pour plusieurs raisons : outre un risque vital non négligeable, restent la difficulté de trouver des donneurs compatibles, la nécessité d'une infrastructure médicale importante et le prix de revient élevé. La méthode est, de ce fait, peu applicable dans la plupart des pays d'origine, où la prévalence est maximale, et la recherche s'oriente vers l'étude pharmacologique de produits susceptibles de stimuler la synthèse d'hémoglobine fotale $(\mathrm{Hb}$ F) par divers mécanismes : induction directe de l'expression des gènes $\gamma$ globine ou régénération érythroïde. L'hydroxyurée (HU) est un produit de cette deuxième catégorie. Agent cytotoxique spécifique de la phase $\mathrm{S}$, l'HU permet l'entrée dans la circulation de précurseurs érythroïdes plus jeunes, et donc plus riches en $\mathrm{Hb} \mathrm{F}$. Après des essais préliminaires de phase I, des essais prolongés de phase II en double aveugle sont en cours aux États-Unis. De nombreuses séries plus courtes sont également connues dans le monde. Une augmentation de l'Hb F a maintenant été retrouvée chez presque tous les malades traités, avec cependant d'importantes variations interindividuelles portant surtout sur le temps d'apparition, qui peut varier de 4 à 24 semaines. Une amélioration clinique est aussi constatée; le méca- interprété comme lié à cette $\mathrm{Hb} \mathrm{F}$, qui, dans la drépanocytose, interrompt le processus de polymérisation et, dans les $\beta$-thalassémies, permet la survie d'un nombre accru de précurseurs érythroïdes.

Un travail, présenté récemment dans un colloque spécialisé, a recherché les effets précoces d'un traitement par l'HU chez trois malades drépanocytaires [1]. Tous les résultats obtenus ont été étudiés en cinétique: deux fois avant le début du traitement, puis 2, 4, 12 et 24 semaines après l'instauration de celui-ci. Il est particulièrement intéressant de retrouver des effets pléiotropiques et échelonnés, car la maladie drépanocytaire est extrêmement complexe et les mécanismes intimes des crises drépanocytaires encore mal compris. L'adhérence cellulaire à l'endothélium vasculaire est ramenée à la normale dans un délai de 2 semaines. La réduction de moitié du compte de réticulocytes intervient dans le même laps de temps. D'autres modifications sont plus étalées dans le temps. On note une diminution progressive du nombre des cellules denses $(d>1,112)$; l'augmentation du contenu de la cellule en $\mathrm{K}^{+}$et la diminution du cotransport $\mathrm{K}-\mathrm{Cl}$ se font dans environ les mêmes temps, ce qui est logique car l'élévation de la densité des cellules, liée à l'activation du cotransport $\mathrm{K}-\mathrm{Cl}$, reflète leur déshydratation. La modification du délai de polymérisation, qui mesure la vitesse de polymérisation de l'hémoglobine $\mathrm{S}$ soumise à une désoxygénation brutale, est, elle aussi, très progressive : $0,3 \mathrm{~s}$ en moyenne initialement, 1,9 $\mathrm{s}$ après 4 semaines et $4,0 \mathrm{~s}$ au bout de 24 semaines; cette dernière cinétique est sensiblement la même que celle de l'augmentation de l'Hb F, des cellules $\mathrm{F}$ et des réticulocytes F. Enfin, la mobilité latérale et rotationnelle de la bande 3 et des glycophorines reste anormalement basse, apparemment peu modifiée.

L'ensemble de ces observations reflète sans doute assez bien l'état de déshydratation des cellules, la facilitation de leurs mouvements et la réduction qui en résulte des risques de vaso-occlusion. Il permet aussi un schéma de classement des effets de l'hydroxyurée en deux groupes, précoces et tardifs, dont la limite se situerait aux environs de 4 semaines après le début du traitement, date avant laquelle aucune augmentation de l'Hb F n'est perceptible. Les effets précoces sont vraisemblablement liés à l'action de HU sur la synthèse des réticulocytes dont on a montré qu'ils étaient anormaux, possiblement responsables de la majeure partie des troubles cliniques [2]. Ce travail montre, enfin, les différents paramètres qu'il serait légitime d'étudier pour apprécier la valeur d'un abord thérapeutique de la drépanocytose.

D.L.

1. Bridges $K$. Early changes in sickle RBC: parameters in patients treated with hydroxyurea. The 19th Annual Meeting. The National Sickle Cell Disease Program. New York City, March 23-26, 1994.

2. Bookchin RM, Ortiz OE, Lew Vl. Evidence for a direct reticulocyte origin of dense red cells in sickle cell anemia. J Clin Invest $1991 ; 87$ : 113-24. 